\title{
The Sun's Smaller Cousins are Running the Universe - The Masses of Red and Brown Dwarfs
}

\author{
Todd J. Henry \\ Department of Physics and Astronomy, Georgia State University, Atlanta GA, USA
}

\begin{abstract}
Although not one red dwarf can be seen with the naked eye, they dominate the solar neighborhood, accounting for no less than $70 \%$ of all stars. The large numbers of these wee denizens of the night actually translate into a surprisingly large amount of mass - in fact, more mass is found in M dwarfs than in any other stellar spectral type.

To determine just how important red dwarfs are to the nature of the Universe, an accurate mass-luminosity relation (MLR) must be determined so that a relatively easily determined characteristic, luminosity, can be converted into the critical parameter, mass. Results from a decade-long observational effort to calibrate the MLR for red dwarfs using the Fine Guidance Sensors (FGSs) on the Hubble Space Telescope (HST) will be highlighted. For many of the binary systems targeted, the interferometric data from HST are combined with radial velocity data to further improve the mass measurements, which often have errors less than $5 \%$. Related results from a large southern sky parallax program to determine accurate distances to red dwarfs, and a search for companions orbiting them, will also be discussed.

The state of mass determinations for M dwarfs' smaller cousins, the $\mathrm{L}$ and $\mathrm{T}$ dwarfs, will also be reviewed. Although we do not yet know the size of the true population of these (primarily) substellar objects, only by mapping out the interplay of their masses and luminosities (which change drastically with time) can we understand their contribution to the mass budget of the Universe.
\end{abstract}

\section{Discussion}

JuAN ECHEVARRIA: With so many new M dwarf masses derived from observations, what improvements have been made on the $\mathrm{M}-\mathrm{R}$ relation?

HENRY: We are using the CHARA Array to measure directly the radii of $\mathrm{M}$ dwarfs. We find that between 0.5 and $0.7 \mathrm{R}_{\odot}$, the models do not fit the data well. Between 0.2 and $0.5 \mathrm{R}_{\odot}$, all seems to be well. There is clearly more observational work to be done, but our first-order improvements in the $\mathrm{M}-\mathrm{R}$ relation indicate that there is quite a bit of understanding yet to be had.

HAns ZinneckeR: How many of your $\mathrm{M}$ stars in the 'Hood actually live in binary systems (i.e., what is the binary fraction)?

HENRY: Of M dwarf systems, $\sim 30 \%$ are multiples. 